\title{
Mapping the physical and chemical properties of the planetary nebula NGC 3242
}

\author{
Hektor Monteiro, Denise Gonçalves, Marcelo Leal-Ferreira, \\ Romano Corradi and Sebastian Sánchez
}

Departamento de Física e Química, Universidade Federal de Itajubá email: hektor.monteiro@gmail.com

\begin{abstract}
We present optical integral field spectroscopy analysis of the main components, with the exception of the halo, as well as of the detected small-scale structures of the planetary nebulae NGC 3242. The observations were obtained with the VIMOS instrument attached to VLTUT3. Spatially resolved maps of the electronic density $\left(N_{\mathrm{e}}\right)$, temperatures $\left(T_{\mathrm{e}}\right)$ and chemical abundances, i.e., in a pixel to pixel fashion of the small and large-scales structures of this planetary nebula are determined in this work. These diagnostic and abundance maps represent important constraints for future detailed three dimensional photoionization modeling of the nebula, as well as providing important information on biases introduced by traditional slit observations.
\end{abstract}

Keywords. planetary nebulae: general, planetary nebulae: individual (NGC 3242)

\section{Introduction}

Planetary nebulae are end products of the evolution of stars with masses below $8 M_{\odot}$ and as such have great importance in many fields in astrophysics, from basic atomic processes in solar like stars to distant galaxies. Although the general picture of planetary nebulae formation is well understood (Kwok 2008), many questions remain unsolved, such as the mechanism by which the stellar ejecta end up forming the many observed morphologies.

Material at different positions within a PN (or different PN components) could be the result of distinct mass-loss episodes of the progenitor star and so could trace the chemical inhomogeneities in the original outflows. So far, this kind of study has been performed for several PNe, using long-slit optical spectroscopy. The results for about $20 \mathrm{PNe}$ (most Type I) are: a)most are chemically homogeneous and b)a few (NGC 2440, K 4-55) show significant abundance variations from one component to another (Gonçalves et al. 2003).

\section{Results}

The observations obtained with VIMOS-IFU instrument attached to VLT-UT3 were reduced with VIMOS pipelines. One important limitation of the observation was the lack of good quality standard star observation, thus due to poor weather, usual flux calibration was not possible. Data were calibrated with respect to a long slit spectra of the object obtained in a posterior date kindly provided by R. Costa.

The reduced line maps show many problems in different quadrants of the IFU. Some problems could be corrected but some could not be removed. To improve the quality of the final maps we applied a FFT filter to remove some of the noise present, especially in the low signal to noise regions. Areas with a signal to noise below 5 were completely removed being set to zero. 
Table 1. Final abundances obtained from integrated line fluxes compared to those obtained by Tsamis et al. (2004).

\begin{tabular}{ccccc}
\hline & This Work & \multicolumn{3}{c}{ Tsamis 04 } \\
\hline Element/H & ICF & Abundance & ICF & Abundance \\
\hline $\mathrm{S} / \mathrm{H}$ & 2.83 & $1.6 \times 10-6$ & 3,52 & $2.4 \times 10-6$ \\
$\mathrm{Ar} / \mathrm{H}$ & 1.18 & $1.6 \times 10-6$ & 1,01 & $9.8 \times 10-7$ \\
$\mathrm{~N} / \mathrm{H}$ & 69.87 & $3.6 \times 10-5$ & 1,53 & $3.4 \times 10-5$ \\
$\mathrm{O} / \mathrm{H}$ & 1.03 & $2.3 \times 10-4$ & 1,17 & $3.3 \times 10-4$ \\
$\mathrm{He} / \mathrm{H}$ & & 0,1 & & 0,1 \\
\hline
\end{tabular}
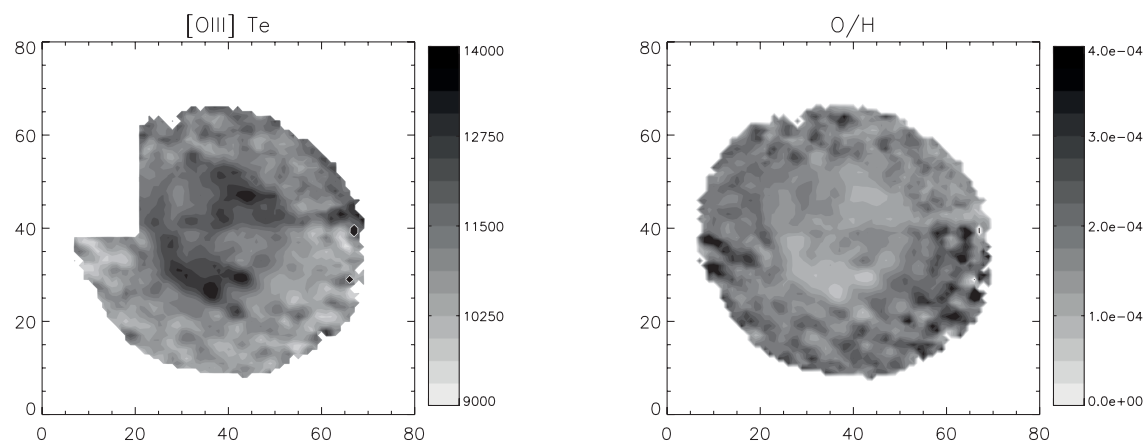

Figure 1. Example of diagnostic and abundance maps derived showing electron temperature obtained from forbidden oxygen lines and $\mathrm{O} / \mathrm{H}$ abundance map obtained from the line maps.

To obtain the final total abundances in cases where a line was not observed we generated a map with emission line intensities obtained from the literature and assumed uniform throughout the the map.

\section{Conclusions}

The results obtained from the resolved IFU observations of NGC3242 are consistent with the ones obtained by Tsamis et al. (2004) within the uncertainties. Considerable structure is seen on the abundance maps but flux calibration uncertainties do not allow us to infer that these are due to actual abundance variations in the nebula. Interestingly, the diagnostics as well as in the abundances determined from the integrated line intensities are in agreement with other literature values when uncertainties are considered. The main lesson is perhaps that, for spatially resolved studies of Pne, more attention has to be given to the flux calibrations and getting extremely good signal-to-noise ratios to ensure precise results and the possibility to detect spatial abundance variations, if they indeed exist.

\section{Acknowledgements}

The author would like to thank CNPq grant 573648/2008-5 and the IAU Grants for financial support.

\section{References}

Gonçalves, D. R., Corradi, R. L. M., Mampaso, A., \& Perinotto, M. 2003, ApJ, 597, 975

Kwok, S. 2008, IAU Symposium, 252, 197

Tsamis, Y. G., Barlow, M. J., Liu, X.-W., Storey, P. J., \& Danziger, I. J. 2004, MNRAS, 353, 953 